\title{
Perspectiva dos pais sobre as consequências funcionais do Transtorno do Desenvolvimento da Coordenaçáo (TDC): revisão da literatura ${ }^{1}$
}

\author{
Beatriz de Arruda Pereira Galváo ${ }^{a}$, Mariana Pereira Veloso ${ }^{a}$, \\ Lívia Paula de Freitaa Carvalho ${ }^{\mathrm{b}}$, Lívia de Castro Magalhães ${ }^{\mathrm{b}}$ \\ anniversidade Federal de Minas Gerais - UFMG, Belo Horizonte, MG, Brasil \\ ${ }^{\mathrm{b}}$ Curso de Terapia Ocupacional, Universidade Federal de Minas Gerais - UFMG, Belo Horizonte, MG, Brasil
}

\begin{abstract}
Resumo: As dificuldades de crianças com Transtorno do Desenvolvimento da Coordenação geralmente são percebidas primeiramente pelos pais durante a realização de atividades que fazem parte da rotina diária de seus filhos. Embora a discussão sobre o impacto funcional do TDC tenha sido ampliada em alguns países, no Brasil esse tema ainda é incipiente. O objetivo deste estudo foi revisar a literatura que descreve a percepção dos pais sobre o impacto do TDC no cotidiano da criança e da família. Realizou-se levantamento das evidências disponíveis na literatura por meio de busca eletrônica nas principais bases de dados, tendo sido usados como palavras-chave os termos comumente adotados pelos pesquisadores e profissionais que trabalham com crianças com TDC. Os critérios para inclusão dos artigos foram uso de metodologia qualitativa, trabalhos publicados no período de janeiro de 1995 a fevereiro de 2012, dados coletados por meio de entrevistas com pais e/ou cuidadores e que se referiam a crianças com sinais específicos de TDC. Foram localizados 594 artigos sobre o transtorno motor, sendo apenas oito qualitativos e todos em inglês. Os dados obtidos nesses artigos evidenciam a preocupação dos pais quanto ao processo de socialização de suas crianças com TDC e a falta de preparo do sistema educacional para lidar com as dificuldades características desse transtorno motor. Conclui-se ser imprescindível, especialmente para profissionais de reabilitação que lidam com crianças com TDC, considerar como os pais percebem as dificuldades de coordenação motora de seus filhos, a fim de desenvolver serviços e ações adequados à realidade das famílias brasileiras.
\end{abstract}

Palavras-chave: Transtorno do Desenvolvimento da Coordenação (TDC), Incoordenação Motora, Disgrafia, Percepção dos Pais.

\section{The perspective of parents on the functional consequences of Developmental Coordination Disorder (DCD): a literature review}

\begin{abstract}
In general, the difficulties presented by children diagnosed with Developmental Coordination Disorder (DCD) are first noticed by parents in the daily routine. Although the discussion about the functional impact of DCD is expanding in some countries, it is still poorly explored in Brazil. The purpose of this study was to review the literature that describes the perception of parents concerning the impact of DCD on the daily life of these children and their families. An electronic search for the evidence available in the literature was conducted in major databases using, as keywords, the terms commonly adopted by researchers and practitioners working with children with DCD. The inclusion criteria were articles that used qualitative methodology, studies published from January 1995 to February 2012, and data collected through interviews with parents and/or caregivers, which described children with specific signs of DCD. Of the 594 articles found, only eight used qualitative methodology - all in English. The data presented in these articles show the concern of parents regarding the socialization process of their children with DCD and the lack of preparation of the educational system to deal with the motor difficulties that characterize
\end{abstract}

Autor para correspondência: Lívia de Castro Magalhães, Escola de Educação Física, Fisioterapia e Terapia Ocupacional, Universidade Federa de Minas Gerais, Campus Pampulha, Av. Antônio Carlos, 6627, CEP 31270-901, Belo Horizonte, MG, Brasil, e-mail: liviam@ gcsnet.com.br Recebido em 16/9/2012; Revisão em 7/4/2013; Aceito em 15/4/2013. 
this disorder. In conclusion, it is essential, especially for rehabilitation professionals who deal with children with DCD, to value how parents perceive the motor coordination difficulties of their children in order to develop actions and services that are adequate to the reality of Brazilian families.

Keywords: Developmental Coordination Disorder (DCD), Coordination impairment, Dysgraphia, Parental Perception.

\section{Introdução}

Crianças com dificuldades na realização de tarefas rotineiras que requerem habilidades motoras, como escrever, jogar bola ou andar de bicicleta, podem ter problemas específicos de coordenação motora. Tais problemas, denominados Transtornos do Desenvolvimento da Coordenação (TDC), caracterizam-se por prejuízo acentuado no desenvolvimento das habilidades motoras na ausência de problemas físicos/neurológicos conhecidos (AMERICAN..., 2002).

De acordo com a terminologia proposta pela Classificação Internacional de Funcionalidade, Incapacidade e Saúde - CIF (ORGANIZAÇÃO..., 2003), o indivíduo com TDC pode apresentar, no componente estrutura e função do corpo, problemas de planejamento motor e declínio do desempenho com a repetição (CERMAK; GUBBAY; LARKIN, 2002). No componente atividade, observa-se lentidão no autocuidado e qualidade precária em habilidades como a escrita. Já na participação verifica-se impacto no desempenho acadêmico, com lentidão e desinteresse tanto nos trabalhos escolares como no envolvimento em jogos e brincadeiras típicas para a idade (MISSIUNA et al., 2006a; SUMMERS; LARKIN; DEWEY, 2008).

Com prevalência de $6 \%$ estimada internacionalmente (AMERICAN..., 2002) e de 4,3\% (CARDOSO, 2011) entre crianças brasileiras em idade escolar, o TDC merece atenção especial por ser uma condição frequente, cujas manifestações podem afetar negativamente o cotidiano das crianças e de suas famílias. Embora alguns países tenham ampliado a discussão sobre o impacto funcional do TDC nas relações sociofamiliares (MISSIUNA et al., 2007), no Brasil a situação é crítica, uma vez que o transtorno e suas características continuam sendo fenômenos pouco explorados (LACERDA; MAGALHÂES; REZENDE, 2007; SILVA; DANTAS; CATTUZZO, 2006). Apesar da alta prevalência, observa-se que o significado do transtorno da coordenação motora no ambiente escolar e familiar ainda é pouco conhecido e, consequentemente, visto com menor importância quando comparado a outros transtornos do desenvolvimento (FERREIRA et al., 2006).
Embora a participação da família seja decisiva para o êxito do processo terapêutico, pouco se sabe acerca da perspectiva de famílias brasileiras sobre os problemas de suas crianças com TDC. Se cada família apresenta suas próprias crenças e valores, desconsiderá-los pode conduzir ao fracasso do tratamento e à frustração. Dessa forma, considerando a importância de se conhecer e valorizar a perspectiva dos pais sobre o transtorno da coordenaçáo, o objetivo deste estudo foi revisar a literatura e levantar os estudos que abordam a percepção dos pais sobre o impacto do TDC no cotidiano da criança e da família. O foco das buscas foram estudos qualitativos que descrevem, de maneira mais explícita, como os pais e as crianças lidam com o TDC.

\section{Metodologia}

Esta revisão teve início com a análise de 476 artigos, localizados por meio de busca sistemática da literatura sobre TDC, publicada entre janeiro de 1995 e julho de 2008, incluídos por Magalhães, Cardoso e Missiuna (2011) em revisão de literatura sobre as limitaçôes nas atividades e restriçôes na participação de crianças com TDC. Como descrito por Magalhães, Cardoso e Missiuna (2011), a busca foi ampla, todos os artigos foram codificados e classificados quanto ao tipo de metodologia do estudo e dentre eles foi possível localizar sete estudos qualitativos que atendiam aos critérios de inclusão e exclusão estabelecidos. Em seguida deu-se continuidade à busca por referências mais recentes, publicadas entre agosto de 2008 e fevereiro de 2012, mantendo-se a mesma estratégia metodológica, localizando-se outros 118 artigos sobre TDC, sendo um qualitativo.

Dessa forma, foram localizados 594 artigos nas duas buscas bibliográficas e apenas oito trabalhos apresentavam dados relacionados à percepçáo dos familiares sobre esse transtorno motor.

\section{Estratégia de busca}

Foi feito levantamento das evidências disponíveis na literatura nacional e internacional por meio de busca eletrônica nas bases de dados MEDLINE, ERIC e LILACS. As palavras-chave para a 
busca foram termos comumente utilizados pelos pesquisadores e profissionais que trabalham com crianças com TDC: clumsy, Developmental Coordination Disorder (DCD), motor skill disorder, incoordination; motor-impairment, dysgraphia and parents perception, Transtorno do Desenvolvimento da Coordenação (TDC), incoordenação motora, disgrafia e percepção dos pais. Os critérios de inclusão de artigos foram: (a) uso de metodologia qualitativa, (b) trabalhos publicados nos idiomas inglês ou português no período de janeiro de 1995 a fevereiro de 2012, (c) trabalhos com dados coletados por meio de entrevistas com pais e/ou cuidadores, e (d) trabalhos que se referiam a crianças com sinais específicos de TDC. Foram excluídos trabalhos nos quais: (a) os participantes apresentavam condiçôes de saúde que impediam o diagnóstico de TDC (ex.: paralisia cerebral, síndromes genéticas, doenças neuromusculares, transtorno invasivo do desenvolvimento), e (b) os critérios de inclusão/ exclusão de participantes não foram específicos o suficiente para determinar se a amostra incluiu crianças com outras condiçóes médicas.

Os artigos localizados foram lidos na íntegra por duas pesquisadoras, de forma independente, e as informaçóes sobre os autores, país de origem e objetivos dos estudos foram organizadas em planilha Excel. Em seguida foi feito resumo dos pontos mais importantes de cada artigo, com o objetivo de discutir as evidências disponíveis relacionadas à temática que orientou esta revisão.

\section{Resultados}

Os oito artigos localizados foram escritos no idioma inglês, em todos eles a técnica preferencial para coleta de dados foi a entrevista, com uso da abordagem fenomenológica como pano de fundo para análise dos relatos obtidos.

Os estudos abordam a percepção e/ou sentimentos dos pais de crianças com sinais de ou já diagnosticadas com TDC sobre suas rotinas diárias, com ênfase nos ambientes doméstico e escolar. Apesar de alguns estudos não apresentarem descrição detalhada dos participantes, a maioria dos informantes foram mães de meninos entre seis e 12 anos. Todas as crianças encontravam-se na escola e a maioria tinha sido diagnosticada com TDC, sendo que, dessas, apenas 13,5\% frequentavam algum tipo de programa terapêutico.

No Tabela 1 encontra-se um resumo de cada estudo localizado. A maioria dos estudos foi realizada no Canadá (75\%), com amostras variando de oito a 87 pais. Observou-se que a preocupação mais frequente entre os entrevistados estava relacionada ao processo de socialização das crianças com TDC, seguida por relatos de despreparo do sistema educacional para lidar com as dificuldades características do transtorno motor. Descrições sobre o comportamento motor das crianças e relatos de restrições na participação em atividades típicas da infância também foram frequentes nos artigos revisados.

\section{Discussão}

Dentre os achados, a principal preocupação dos pais esteve relacionada com a vida social de suas crianças (MANDICH; POLATAJKO; RODGER, 2003; MISSIUNA et al., 2006b) e a possibilidade de estigmatização, devido ao fato de a dificuldade motora restringir a participação em determinadas atividades, o que pode conduzir à rejeição pelos pares (SEGAL et al., 2002). Devido aos déficits de coordenação motora, as crianças com TDC geralmente são as últimas a serem escolhidas para participar dos jogos e atividades esportivas e muitas se tornam alvo de bullying dos colegas (MANDICH; POLATAJKO; RODGER, 2003). A maioria dos pais entrevistados relatou que o fracasso é uma experiência comum para suas crianças (AHERN, 2000), enquanto outros afirmaram preocuparem-se quanto às "escolhas estranhas" feitas por suas crianças na tentativa de amenizar a inabilidade em participar de determinadas atividades como, por exemplo, jogar bola. Muitas delas, ao se tornarem conscientes de suas limitaçóes, preferem brincar sozinhas, ou então buscam colegas mais novos, o que, por sua vez, limita as oportunidades de praticarem suas habilidades e interagirem socialmente com os colegas da sua faixa etária (SEGAL et al., 2002).

Foi observado que a jornada dos pais na tentativa de entender seus filhos com TDC é repleta de incertezas (AHERN, 2000; PLESS et al., 2001). Alguns deles notam, por exemplo, que a criança evita atividades que envolvem coordenação motora, tem dificuldade no aprendizado de novas habilidades e se cansa com facilidade, mas não identificam isso, necessariamente, como um problema (MISSIUNA et al., 2006c). Alguns entrevistados expressaram dúvida quanto a atribuir as dificuldades da criança a diferenças individuais (i.e., variação normal da habilidade) ou a um problema clínico que requer intervenção específica (MISSIUNA et al., 2007).

A busca por respostas para tais incertezas é marcada principalmente pelo ingresso na escola, pois a partir desse momento os pais passam a ter as outras crianças como ponto de referência para comparação. 


\begin{tabular}{|c|c|c|c|c|c|c|c|c|}
\hline & 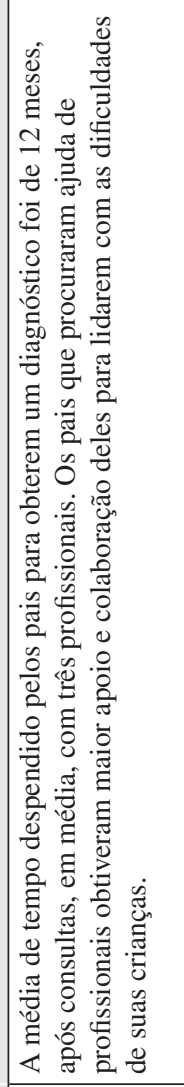 & 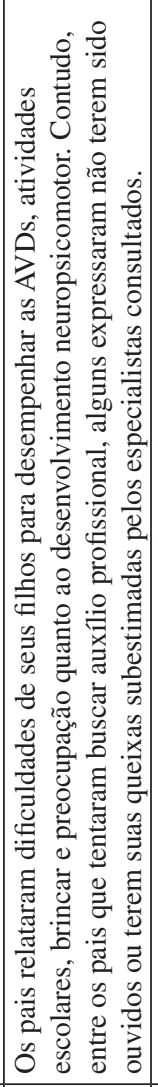 & 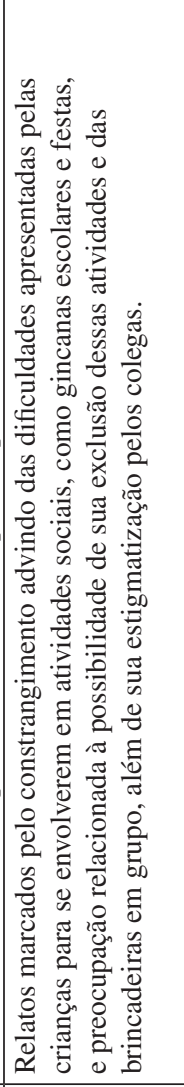 & 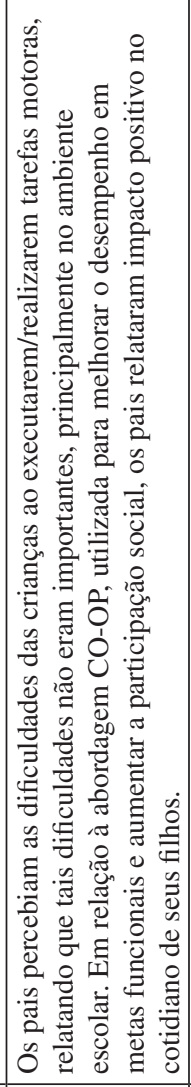 & 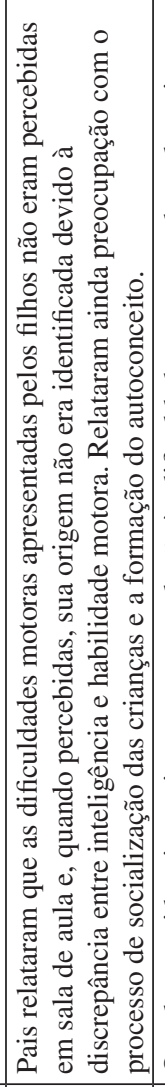 & 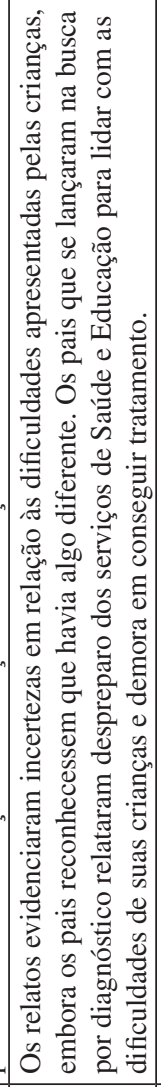 & 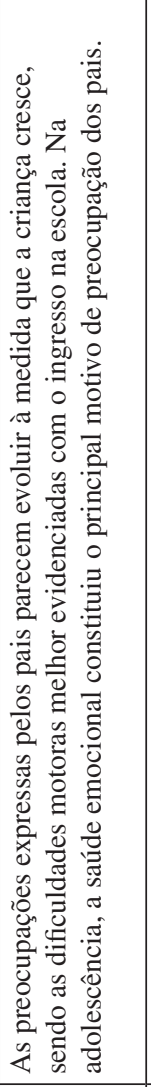 & 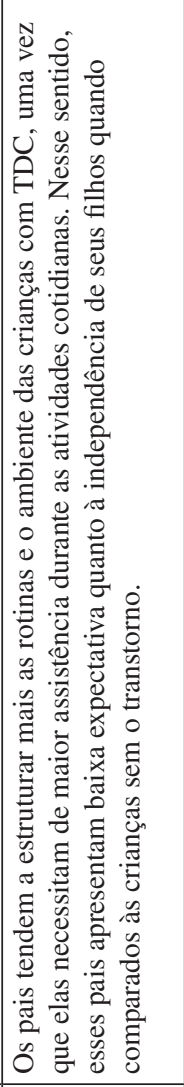 \\
\hline & 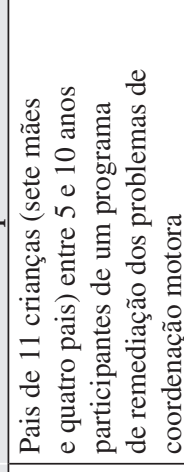 & 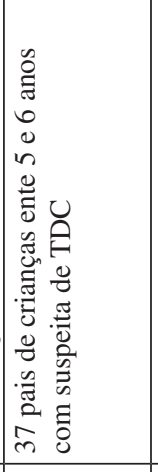 & 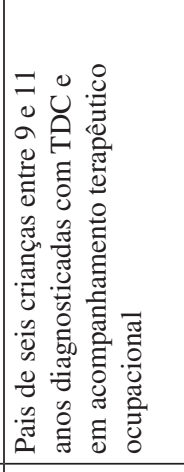 & 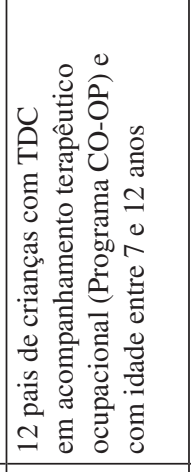 & 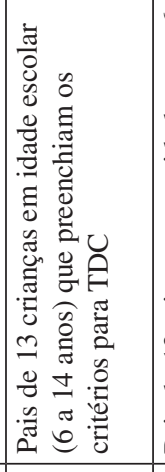 & 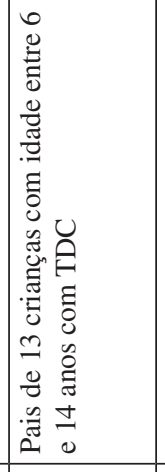 & 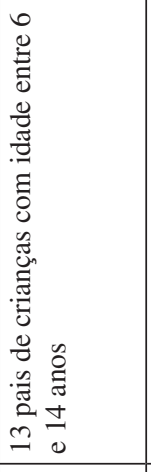 & 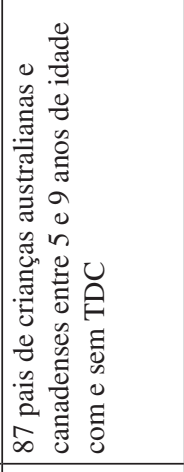 \\
\hline 递 & 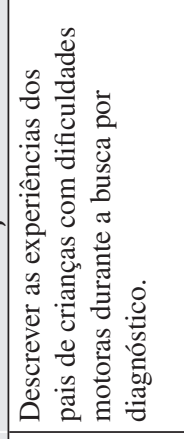 & 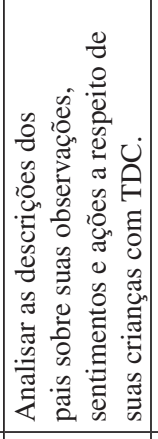 & 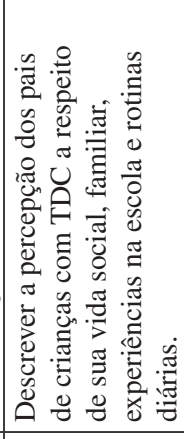 & 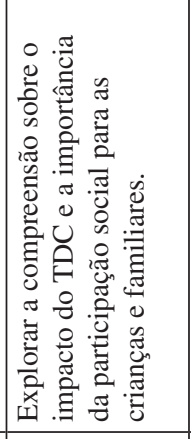 & 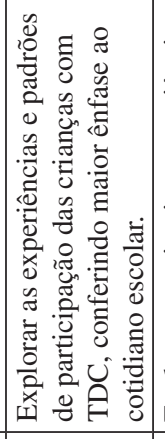 & 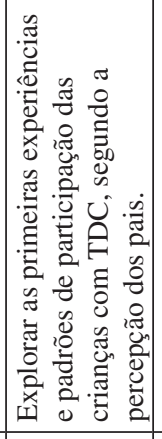 & 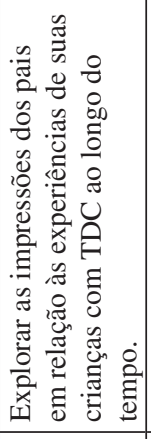 & 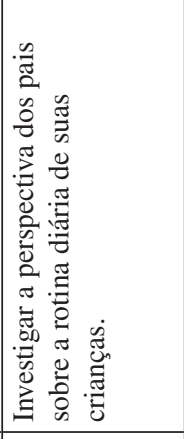 \\
\hline Ðே & 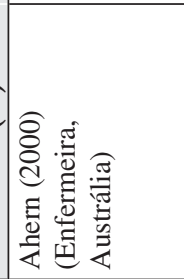 & 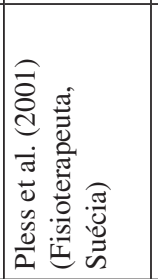 & 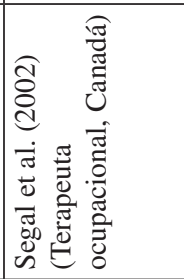 & 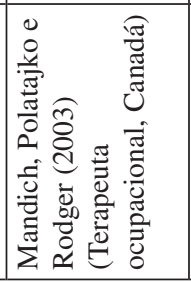 & 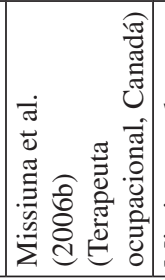 & 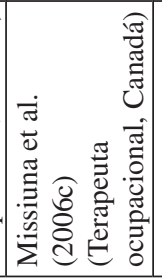 & 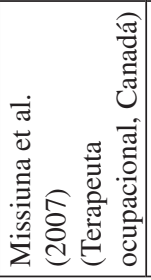 & 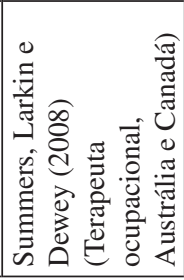 \\
\hline
\end{tabular}


No ambiente escolar, as diferenças nas habilidades motoras, antes vistas como preocupação menor, adquirem novos significados e passam a ser percebidas como dificuldades reais (MISSIUNA et al., 2007). Surgem desafios com a escrita cursiva, que demanda maior tempo e esforço por parte da criança com problemas motores, e com o engajamento em atividades físicas, incluindo esportes, gincanas e brincadeiras no recreio, uma vez que as crianças com TDC têm dificuldade no aprendizado de novas habilidades (MISSIUNA et al., 2006b).

Os relatos mostram que os problemas dessas crianças, muitas vezes, não são considerados importantes, principalmente por alguns educadores, pelo fato de não serem táo evidentes como as questóes enfrentadas pelas crianças cadeirantes e com paralisia cerebral (MANDICH; POLATAJKO; RODGER, 2003). Alguns pais sinalizaram ainda que embora suas crianças fossem consideradas brilhantes, elas tinham dificuldades para fazer as tarefas de sala de aula com independência e para demonstrarem suas habilidades, especialmente por meio do trabalho escrito, além de apresentarem dificuldades para acompanhar a rotina escolar e para organizar seu material (MISSIUNA et al., 2006b).

De acordo com os relatos, problemas na escola geralmente conduzem ao aumento do estresse nas relaçôes familiares, pois ao se depararem com as dificuldades enfrentadas por suas crianças os pais buscam suporte no sistema educacional (MISSIUNA et al., 2006c). Contudo, frequentemente eles se viam sozinhos, sem ajuda da escola para lidar com as dificuldades que observavam (MANDICH; POLATAJKO; RODGER, 2003). Conforme pontuado por Missiuna et al. (2006c), os desafios vivenciados pelos pais de crianças com dificuldades motoras são vários pelo fato de o TDC ainda não ser um diagnóstico bem conhecido dentro do sistema escolar e dos serviços de saúde, surgindo obstáculos que dificultam o acesso à ajuda especializada para essa população.

Além do baixo desempenho das crianças com TDC na rotina escolar, os pais relataram desempenho insatisfatório nas atividades de vida diária (AVDs), que por serem consideradas simples pelas crianças sem TDC, geram sentimento de fracasso e estupidez nas crianças com TDC (MANDICH; POLATAJKO; RODGER, 2003). Por exemplo, os participantes do estudo de Summers, Larkin e Dewey (2008) expressaram que a rotina diária focada nas atividades de autocuidados foi considerada menos flexível pelos participantes, uma vez que as crianças com TDC são lentas ao executarem tarefas básicas, necessitando de ajuda consistente e de ambiente mais estruturado para realizar as tarefas dentro do prazo estipulado. Os pais relataram que crianças com TDC precisam de rotinas mais estruturadas e de assistência para realizar atividades básicas e funcionais, resultando em baixa expectativa quanto a independência no desempenho de tarefas ocupacionais (SUMMERS; LARKIN; DEWEY, 2008).

Considerando a perspectiva da CIF (ORGANIZAÇÃO..., 2003), nota-se mudança nas questóes reportadas nos relatos dos pais. Nas crianças mais jovens, eles se referem a prejuízo na coordenação motora que precede e está relacionado a queixas de problemas no desempenho de atividades, primeiro em casa e depois na escola mas, ao longo do tempo, as questôes relacionadas à participação social adquirem maior relevância (MISSIUNA et al., 2007). Como discutido por Missiuna et al. (2007), parece haver progressão da preocupação com o desempenho motor e o brincar, nos primeiros anos, para os problemas com o autocuidado, dificuldades acadêmicas e sociais em meados da infância, que convergem para preocupação com os desafios relacionados à saúde emocional e relacionamento social no início da adolescência.

\section{Considerações finais}

Os estudos indicam que, apesar das incertezas, os pais percebem que suas crianças têm problemas motores importantes, que se manifestam por dificuldades no desempenho de atividades cotidianas e na participação social. Além disso, os pais relataram preocuparem-se mais com as limitaçôes na participação do que com as dificuldades motoras em si, apontando que o relacionamento com os colegas e o envolvimento em atividades e situaçóes típicas da faixa etária, tais como jogos e brincadeiras em grupo, têm um valor especial para a criança.

É imprescindível, portanto, que profissionais de reabilitação que lidam com crianças com TDC valorizem as percepçóes dos pais acerca das dificuldades de coordenação motora de suas crianças, procurando entender o significado que esses problemas têm para os pais e as estratégias que utilizam para sua superação. Contudo, não basta apenas considerá-los como fontes válidas de informação a respeito do nível de habilidades e dificuldades de seus filhos, tampouco escutá-los unicamente no momento da anamnese. É preciso, de fato, acolher e incorporar suas dúvidas, preocupaçóes e valores ao longo de todo o processo terapêutico, desde a definiçáo dos objetivos do tratamento à reavaliação, conferindo espaço e condiçôes para que eles se sintam corresponsáveis pelo tratamento. 
Esta revisão evidenciou a escassez de artigos que abordam a percepção dos pais de crianças com TDC principalmente no cenário nacional. Fato esse que aponta para a necessidade de se fomentar o desenvolvimento de pesquisas, com destaque para as qualitativas, voltadas para essa população, com o objetivo de explorar como os pais brasileiros percebem as dificuldades de coordenaçấo motora de suas crianças e, consequentemente, ampliar o reconhecimento do problema nas áreas de Educação e Saúde. Por ser um transtorno motor discreto, o TDC muitas vezes é ignorado ou passa despercebido, sendo importante recorrer ao relato de pais para entender a dimensão das questốes motoras na vida da criança e de sua família. Acredita-se também que, na medida em que as dúvidas e preocupaçôes dos pais forem acolhidas e incorporadas ao processo terapêutico, os profissionais que lidam com as crianças com TDC poderão desenvolver uma assistência terapêutica de qualidade, congruente com a realidade sociocultural e o contexto familiar.

\section{Referências}

AHERN, K. Something is wrong with my child: a phenomenological account of a search for a diagnosis. Early Education \& Development, London, v. 11, n. 2, p. 187-201, 2000. http://dx.doi.org/10.1207/s15566935eed1102_4

AMERICAN PSYCHIATRIC ASSOCIATION. Manual Diagnóstico e estatístico de Transtornos mentais: DSM-IV-TR. 5th ed. Tradução de M. R. Jorge. Porto Alegre: Artes Médicas, 2002.

CARDOSO, A. A. Validade da avaliação da coordenação e destreza motora-ACOORDEM para crianças de 7 e 8 anos de idade. 2011. 196 f. Tese (Doutorado em Ciências da Reabilitação)-Universidade Federal de Minas Gerais, Belo Horizonte, 2011.

CERMAK, S.; GUBBAY, S.; LARKIN, D. What is developmental coordination disorder? In: CERMARK, S. A.; LARKIN, D. Development coordination disorder. Columbia Circle: Delmar, 2002. p. 2-22.

FERREIRA, L. F. et al. Desordem da coordenação do desenvolvimento. Motriz: revista de educaçáo física, Rio Claro, v. 12, n. 3, p. 283-292, 2006.

LACERDA, T. T. B.; MAGALHÃES, L. C.; REZENDE, M. B. Validade de conteúdo de questionários de coordenaçáa motora para pais e professores. Revista de Terapia Ocupacional da USP, São Paulo, v. 18, n. 2, p. 63-77, maio/ago. 2007. http://dx.doi.org/10.11606/ issn.2238-6149.v18i2p63-77

MAGALHÃES, L. C.; CARDOSO, A. A.; MISSIUNA, C. Activities and participation in children with developmental coordination disorder: A systematic review. Research in Developmental Disabilities, New York, v. 32, p. 1309-1316, 2011. PMid:21330100. http://dx.doi.org/10.1016/j. ridd.2011.01.029
MANDICH, A. D.; POLATAJKO, H. J.; RODGER, S. Rites of passage: Understanding participation of children with developmental coordination disorder. Human Movement Science, Amsterdam, v. 22, p. 583-595, 2003. http://dx.doi.org/10.1016/j.humov.2003.09.011

MISSIUNA, C. et al. "Missed and Misunderstood": Children with coordination difficulties in the school system. International Journal of Special Education, California, v. 21, n. 1, p. 53-67, 2006 a.

MISSIUNA, C. et al. Mysteries and Mazes: Parent's experiences of children with developmental coordination disorder. Canadian Journal of Occupational Therapy, Toronto, v. 73, n. 1, p. 7-17, 2006b. PMid:16570837. http://dx.doi.org/10.2182/cjot.05.0010

MISSIUNA, C. et al. Parental questions about developmental coordination disorder: A synopsis of current evidence. Pediatrics Child Health, Oxford, v. 11, n. 8, p. 507-512, 2006c. PMid:19030319.

MISSIUNA, C. et al. A Trajectory of Troubles: Parent's Impressions of the Impact of Developmental Coordination Disorder. Physical \& Occupational Therapy in Pediatrics, London, v. 27, n. 1, p. 81-101, 2007. PMid:17298942. http://dx.doi.org/10.1080/J006v27n01_06

ORGANIZAÇÃO MUNDIAL DE SAÚDE - OMS. CIF: Classificação Internacional de Funcionalidade, Incapacidade e Saúde. São Paulo: EdUSP, 2003.

PLESS, M. et al. Children with Developmental Cooordination Disorder: A Qualitative Study of Parents' Descriptions. Advances in Physiotherapy, London, v. 3, p. 128-135, 2001. http://dx.doi. org/10.1080/140381901750475375

SEGAL, R. et al. Stigma and its management: A pilot study of parental perceptions of the experiences of children with developmental coordination disorder. American Journal of Occupational Therapy, New York, v. 56, p. 422-428, 2002. http://dx.doi.org/10.5014/ajot.56.4.422

SILVA, J. A. O.; DANTAS, L. E.; CATTUZZO, M. T. Teste mabc: aplicabilidade da lista de checagem na região sudeste do Brasil. Revista Portuguesa de Ciências do Desporto, Lisboa, v. 6, n. 3, p. 356-361, 2006.

SUMMERS, J.; LARKIN, D.; DEWEY, D. What Impact does Developmental Coordination Disorder have on Daily Routines? International Journal of Disability, Development and Education, Queensland, v. 55, n. 2, p. 131-141, 2008. http://dx.doi.org/10.1080/10349120802033485

\section{Contribuição dos Autores}

Beatriz de Arruda Pereira Galvão: concepção do texto, organizaçáo de fontes e redação do texto. Mariana Pereira Veloso e Lívia Paula de Freitas Carvalho: busca e revisão dos artigos, redação do texto. Lívia de Castro Magalhães: concepção, redação e revisão do texto. Todas as autoras aprovaram a versão final do texto. 


\section{Notas}

${ }^{1}$ Apoio: Auxílio pesquisa do Conselho Nacional de Pesquisa e Desenvolvimento Científico (CNPq) e da Fundação de Amparo à Pesquisa de Minas Gerais (Fapemig). 